\title{
Importance of Clinical Evaluation of Language Fundamental (CELF-2) as an Aid for Treatment Planning
}

\author{
Swetha Grandhi ${ }^{1}$, Srilalitha Parameswaran ${ }^{2}$, Stephy Varghese $^{3}$ \\ ${ }^{1}$ Lecturer, Department of Speech Language Studies, Dr. S.R. Chandrasekhar Institute of Speech and Hearing \\ Lingarajapuram, Hennur Main road, Bangalore-560084, Karnataka, India
}

\begin{abstract}
${ }^{2}$ Graduate Clinician², Department of Speech Language Studies, Dr. S.R. Chandrasekhar Institute of Speech and Hearing, Lingarajapuram, Hennur Main road, Bangalore-560084, Karnataka, India

${ }^{3}$ Graduate Clinician, Department of Speech Language Studies, Dr. S.R. Chandrasekhar Institute of Speech and Hearing, Lingarajapuram, Hennur Main road, Bangalore-560084, Karnataka, India
\end{abstract}

\begin{abstract}
Language assessment provides the back bone for intervention program. Assessment guides diagnosticians in collecting reliable and valid information, integrating and interpreting the information to make a judgment or a decision. There are various language assessment tools for children with hearing impairment. But there are very few tools that assess the child in a holistic perspective. Hence the current study aims to attempt on one such assessment tool CELF Preschool-2 which helps the diagnosticians to plan for the treatment in a precise way. A 6 year male child post cochlear implant was accompanied with his parents to the department of speech and language studies for re-evaluation. Detailed speech and language assessment revealed age appropriate speech and language skills. CELF Preschool-2was administered to evaluate and pinpoint varied components of language (Phonology, morphology, syntax, semantics and pragmatics) for appropriate rehabilitation. Results revealed that child has mild to moderate language deficits across the various subtests. Thus it can be concluded that multiperspective language assessment helps the clinicians to plan specific rehabilitation services for individuals with hearing impairment.
\end{abstract}

Keywords: Language, Assessment, Holistic, Language, Assessment, Holistic

\section{Introduction}

Language assessment is a complex endeavour. Assessment is the process of collecting reliable and valid information, and integrating and interpreting the information to make a judgement or a decision. (Kenneth and Shipley, 1992).The outcome of assessment is diagnosis, which is the clinical decision regarding the presence or absence of a disorder.

According to Rhea Paul (2007), during the process of an assessment, as a speech language pathologist our aim should focus on the identification of the strength and weakness of the client, arrive at an appropriate diagnosis and the severity of the problem and plan for structured treatment.

SubbaRao(1992) stated three primary justifications for assessment; the first is to identify children with language problems, second is to establish baseline function and finally evaluate the progress in order to assess the effectiveness and the efficiency of the intervention program.

The term oral language is used to assess the language abilities of hearing children to distinguish from written language. Given the visual-gestural modality of the language of many deaf individuals, it is more appropriate to refer as face-to-face language.

The assessment process provides the back bone for intervention program. Communication and language assessment is a systemic approach to measure and evaluate a child's skills, capabilities and limitations in communication.
This can be achieved through tests of various kinds, careful recording and observation of general and more specific skills.

The communication assessment is performed not only to develop individual remedial programs and to monitor a child's progress, but also to help the professionals gain insight into the communication problems of hearingimpaired children. As a speech language pathologist performing in depth language assessment for a child with hearing impairment is a complex endeavor due to the challenges that a diagnostician

Faces during the process of assessing the child in a holistic perspective. Assessment of child in a holistic view includes the assessment of child's language in terms of phonology, morphology, syntax, semantics and pragmatics. There are various standardized tests that measure the language in terms of all these domains individually. But there are very few tests that assess these components in a holistic perspective. Hence assessment of child in a holistic view is essential for planning specific intervention strategies.

\section{Literature Survey}

Ann et al(2008) assessed spoken language score of five children using cochlear implant compared to hearing age males at school using standardized tests (clinical evaluation of language fundamental). These children (mean age 5 years and 10 months) attended programs in the united states focusing on the use of auditory- oral educational approach. 


\section{International Journal of Science and Research (IJSR) \\ ISSN (Online): 2319-7064}

Index Copernicus Value (2013): 6.14 | Impact Factor (2014): 5.611

Results revealed that age appropriate scores in 50\% of children on receptive vocabulary ,58\% on expressive vocabulary, $46 \%$ on verbal intelligence, $47 \%$ on receptive and $39 \%$ on expressive language. Regression analysis indicated that the children who received the implant at younger ages had higher scores on all language tests than older implanted children on averages, children with cochlear implant performed better on certain language measures than others, indicating that some areas of language will be more difficult for these children to master than others.

Gretchen et al (2002) grounded their areas of research in assessing receptive and expressive language skills of 7 children with five years of expressive using a cochlear implant. Language skills of these children was assessed using standardised tests such as clinical evaluation of language fundamental-3, pea body picture vocabulary testrevised, and the expressive vocabulary subtest of the test of word knowledge . Results demonstrated impaired skills relative to normal hearing children on one or more subtests. Semantic skills were evident compared to weaker syntactic and morphological abilities.

Spencer (2004) evaluated individual a differences in language performance alter cochlear implantation (one-third years of age) in 13 prelanguage deaf children .Individual post implant language skills ranged from extremely delayed to age appropriate on average, skills varied across domains, on vocabulary, several children functioned in the average range compared with hearing peers, but all were below that range on a test emphasizing syntax (CELF- preschool).

\section{Need for Study}

As there are limited tests that focus to assess the functional language abilities of the client in a multi-perspective view that is in terms of form which include (phonology, morphology, syntax,) content (semantics) and use (pragmatics) .Hence there is a need to understand them to plan for the specific and appropriate treatment program.

\section{Aim of Study}

The aim of the study is to highlight one such test (CELF-Pre School $2^{\mathrm{ND}}$ Edition) that helps the speech language pathologist to plan the treatment in a structured manner.

\section{Method}

A male child aged 6.6 years attending speech and language therapy at our institute since 1 year came for a reevaluation after post Cochlear implant. Prenatal, natal and post natal history reveals no complications. Detailed speech and language evaluation was carried out to assess the speech and language skills of the child (Post implant).

Formal assessment carried by a speech language pathologist to evaluate speech and language skills involved battery of tests such as Receptive Expressive Emergent Language Scale (REELS), COMDEALL (Communication developmental elicits for language learning), Assessment of language development (ALD), Gilman and Gorman Speech and Language Developmental Chart, Bankson Language Screening Test (BLST) and Clinical evaluation of Language Fundamentals( CELF-Pre School 2 Edition ).

\section{Results and Discussion}

Results of detail speech and language assessment revealed receptive and expressive language age of 6-7years in accordance with Receptive Expressive Emergent Language Scale (REELS). Communication development elicits for language learning (COMDEALL) reveals Motor,

Language, Cognition, Social, Emotional skills reached the ceiling level (6years). Assessment of language development (ALD) reveals receptive and expressive language age of 6 to 6.11 years. Gilman and Gorman speech and language developmental chart reveals all the domains (phonology, semantics, play, syntax-morphology, and pragmatics) as age adequate. Assessment of child's morpho-syntactic skills using Bankson Language Screening Test (BLST) obtained a score of 123 out

of 153 suggestive of age adequate skills (6-6.11 years).

According to all the standardized test administered child's receptive and expressive language skills was age adequate. At this point of time multiperspective language assessment should be carried to assess in-depth language skills of child (phonology, morphology, syntax, semantics and pragmatics) as a speech and language pathologist we should perform a multi-perspective assessment that helps the clinicians to pinpoint the child`s strength and weakness on the different domains of language and plan specific intervention targets.

Clinical evaluation of language fundamentals enables the diagnosticians to perform such a specific assessment.

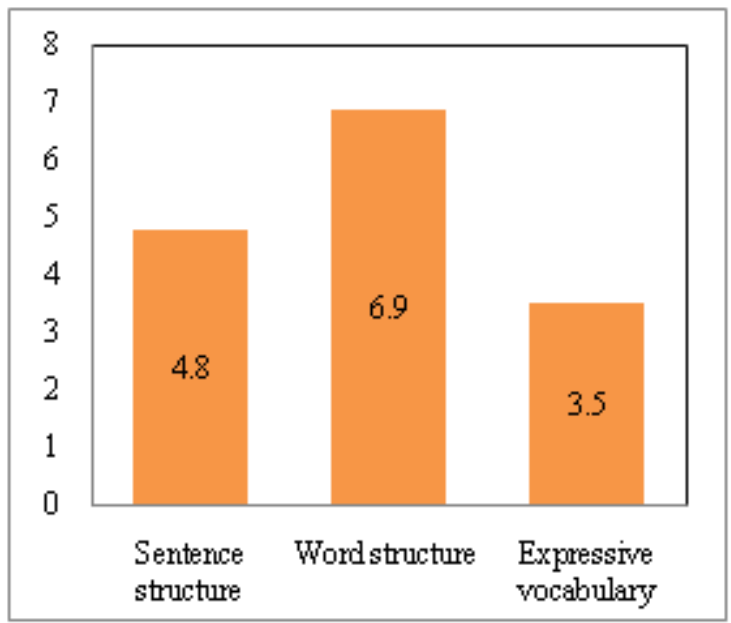

Figure 1: Representation of Core Language scores

From figure 1 one can infer that the result of Clinical evaluation of Language Fundamentals (CELF-Pre School 2nd Edition) reveals that the performance of core language score is low with respect to the subtests sentence structure and expressive vocabulary. But for word structure task the child obtained age equivalence score. 


\section{International Journal of Science and Research (IJSR) \\ ISSN (Online): 2319-7064}

Index Copernicus Value (2013): 6.14 | Impact Factor (2014): 5.611

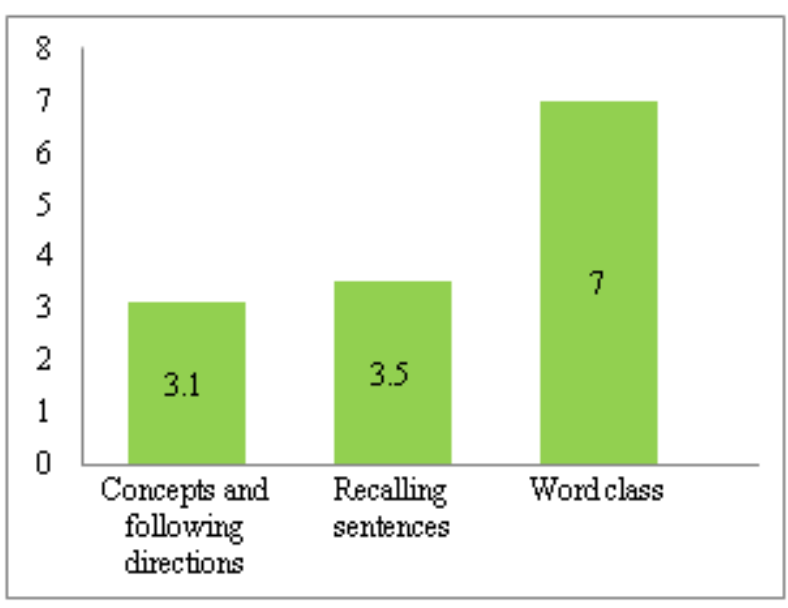

Figure 2: Representation of subtests

Figure 2 depicts that the scores obtained for word class is age adequate. Child performance is poor for recalling sentences, concepts and following directions.

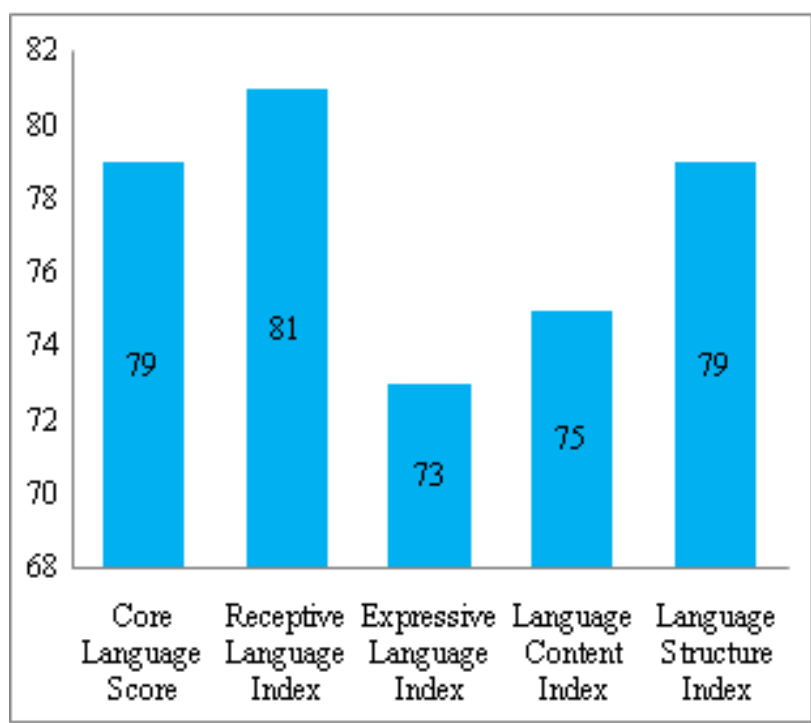

Figure 3: Representation Core and Index language scores

Level 1 and level 2 of CELF Preschool-2 indicating diagnosis and description of language disorder summarizes the Core Language, index, and subtest scores. From figure 3 we can understand that the Core Language score is 79 (confidence interval of 69-89 at the 95\% level), which indicates the performance is low with respect to the subtests sentence structure and expressive vocabulary. But for word structure task the child got an age equivalence score.

The Receptive index scores is 81 (confidence intervals of $70-92$ at the $95 \%$ confidence which indicate performance is low for sentence structure, concepts and following directions. But for word class receptive task child got an age equivalence score. The expressive index scores is 73 (confidence intervals of 65-81) at the 95\% confidence interval which indicate age equivalent performance for word structure task, but for recalling sentence and expressive vocabulary the child obtained a low score.

CELF Preschool-2 summarizes the Language content index and subtest scores. Language content index is 75 (confidence interval of 66-84) at the 95\% level. This score indicates the performance is low with respect to the subtest expressive vocabulary, concepts and following directions. For word class-total the child's score is higher than his age equivalence score. The language structure index scores is 79 (confidence intervals of 69-89) at the 95\% confidence interval which indicate age equivalent performance for word structure task, but for recalling sentence and sentence structure the child obtained a low score.

Level 3 reveals early classroom and literacy fundamentals. This level provides additional information about the client's performance on the supplementary subtest. The child obtained a score of 11 out of 39 indicative of poor performance in recalling sentences in context. A score of 23 on 24 was obtained for phonological awareness skills and thus meets the criteria. Client's parents completed the PreLiteracy Rating Scale and the raw score met the criterion for his age. A score of 17 on 18 was obtained for basic concepts skills and thus meets the criteria.Level 4 deals with evaluating language and communication in context .Additionally, client's parents completed the descriptive pragmatic profile and client's raw score met the criterion for his age.

\section{Conclusion}

Even though language skills are age adequate in children with hearing impaired through formal assessment. They need in depth evaluation of language skills in holistic perspective using multi perspective assessment process which will helps the diagnosticians to pinpoint child's language and communication strengths and weakness and guides the speech language pathologists to establish the base line for intervention program.

\section{References}

[1] Kenneth Shipley, Julie McAfee (2004) Assessment of speech and language pathology- A Resource manual fifth edition. Pg. no: 1-10.

[2] Rhea Paul (2007) Language disorders from infancy to adolescences. Third edition. Pg. no: 213-215.

[3] Subba Rao (1992). Developing communication skills in MR. Pg. no. 135-265.

[4] Elizabeth Carrow - Woolfolk (1982) an integrative approach to Language disorders in children. Grune and Stratton publishers

[5] Spencer PS. Individual differences in language performance after cochlear implantation at one to three years of age: Child, family, and linguistic factors. Journal of Deaf Studies and Deaf Education.2004; 9:395-412.

[6] Anne; Moog, language score of children using cochlear implant compared to hearing age -mates at school entry. Journal of deaf studies and deaf studies; summer2009, vol.14 issue 3, p371.2004.

[7] Clinical evaluation of Language Fundamentals preschool- second edition, Australian and New Zealand Standardized

[8] Elisabeth h. wig, Wayne a. Secord, Eleanor semel (2006) - Clinical evaluation of language Fundamentals Preschool- Second UK Edition. Pearson Publications 


\section{International Journal of Science and Research (IJSR) \\ ISSN (Online): 2319-7064}

Index Copernicus Value (2013): 6.14 | Impact Factor (2014): 5.611

\section{Author Profile}

G. Swetha presently working as a lecturer in the Department of Speech and Language Studies in Dr.S.R. Chandrasekhar Institute of Speech and Hearing, Bangalore, Karnataka, India. I am an active participant in research and also guided students for the research related activities. And my core areas of interest are child language disorders, fluency and voice. I have presented and published many research articles in the National Level Journals and also in the proceedings.

Srilalitha Parameswaran, a graduate student (Bachelors in Speech Language Pathology and Audiology) from Dr.S.R Chandrasekhar Institute of Speech and Hearing Bangalore, Karnataka, India. As a student I am an active participant in research activities.

Stephy Varghese, a graduate student (Bachelors in Speech Language Pathology and Audiology) from Dr.S.R Chandrasekhar Institute of Speech and Hearing Bangalore, Karnataka, India. As a student I am an active participant in research activities. 